\title{
Casos de cisticercose em pacientes internados pelo Sistema Único de Saúde: distribuição no Estado do Ceará
}

\author{
Cysticercosis' admissions in public health hospitals: \\ Ceará State distribuition
}

\author{
Mônica Cardoso Façanha1
}

\begin{abstract}
RESUMO
Estudou-se a distribuição geográfica da cisticercose, sua relação com rebanho suíno e disponibilidade de tomografia computadorizada no município de residência de pacientes registrados no Sistema de Informação Hospitalar do Ceará (19962004). Internaram-se 424 pacientes com cisticercose (neurocisticercose 98,3\%) procedentes de 75 municípios. Inexistiu relação entre a distibuição geográfica dos casos e a existência de tomografia computadorizada ou rebanhos suínos.
\end{abstract}

Palavras-chaves: Neurocisticercose. Cisticercose. Sistemas de informação.

\begin{abstract}
The geographic distribution distribution of case registered in the System of Hospital Information of Ceará (1996-2004) and its relation with flock swine and availability of computerized tomography scan in patients' residence city, from those were studied. 424 Patients were admitted with cysticercosis ( neurocysticercosis 98.3\%) originating from 75 cities. No relation existed between computerized tomography in city of residence or swine flocks and cysticercosis prevelance.
\end{abstract}

Key-words: Neurocysticercosis. Cysticercosis. Information systems.

A cisticercose é doença de notificação compulsória em raros estados e municípios brasileiros, o que contribui para a falta de conhecimento da prevalência dessa entidade no Brasil. A prevalência de epilepsia de início tardio em nossa sociedade é de 29,5\% ${ }^{9}$. Cisticercose relacionada à epilepsia foi encontrada em Florianópolis, SC (19,2\% dos casos iniciados depois dos 18 anos) ${ }^{8}$, Chapecó, SC $(24 \% \text { dos internados })^{10}$, Recife, $\mathrm{PE}(8,8 \%)^{11}$, Mulungu do Morro, BA (15,3\% dos pacientes com anticorpos para neurocisticercose) $)^{5}$. Foi encontrada em 3,3\% das autópsias realizadas em Uberaba, $\mathrm{MG}^{10}$ e em 1,6\% das realizadas no Serviço de Patologia do Hospital das Clínicas da Faculdade de Medicina de Ribeirão Preto?.

A cisticercose pode acometer praticamente todos os órgãos do corpo. No entanto, a neurocisticercose, cujas manifestações clínicas mais freqüentes são cefaléia e convulsões, pode ser confundida com epilepsia idiopática e cefaléia crônica. Atualmente, é diagnosticada com mais freqüência, devido à disponibilização da tomografia computadorizada (TC) de crânio, especialmente para usuários do Sistema Único de Saúde (SUS) . Cefaléia intensa, convulsões e sinais neurológicos focais são indicações paraa TCde crânio ${ }^{8}$. Este estudo teve como objetivo verificar as características demográicicas e evolução clínica durante internação em hospital do Sistema Único de Saúde, além da distribuição dos pacientes por município e sua relação com a disponibilidade de tomografia computadorizada e a criação de suínos.

\section{MATERIAL E MÉTODOS}

Trata-se de estudo descritivo, retrospectivo, em que foram incluídos todos os pacientes residentes no Ceará e internados em hospitais, registrados no Sistema de Informação Hospitalar ( SIH) ${ }^{3}$ do Estado do Ceará, entre 1996 e 2004. Havia exames por tomografia computadorizada disponíveis para pacientes do SUS nos municípios de Barbalha, Fortaleza, Juazeiro do Norte, Sobral e Crato (antes do ano 2000) ${ }^{3}$, Russas e Quixadá (a partir de 2001), Maracanaú (a partir de 2002) e Iguatu (apenas durante 0 ano 2002).

Em 1995, o Ceará possuía 13.000 matrizes de suínos, reduzindo para apenas $6.000^{6} \mathrm{em} 2005$ e 0 consumo de carne suína

\footnotetext{
1. Departamento de Saúde Comunitária da Faculdade de Medicina da Universidade Federal do Ceará, Fortaleza, CE. Endereço para correspondência: Profa Mônica Cardoso Façanha. R. Pinto Madeira 777/701, Centro 60150-000 Fortaleza, CE. Tel: 5585 3251-1549; Fax: 5585 3366-8050

e-mail: mfacanha@yahoo.com

Recebido para publicação em 12/6/2005

Aceito em 11/7/2006
} 
era de $4 \mathrm{~kg}$ por habitante, bem abaixo da média nacional (12kg/ hab/ano). Os municípios onde se localizam as granjas são Cascavel, Caucaia, São Gonçalo do Amarante e Maranguape ${ }^{4}$.

0 Tabwin foi utilizado para a distribuição dos casos por forma e evolução clínica, sexo, idade, município de residência e diagnóstico ${ }^{3}$. A freqüência das variáveis é apresentada em forma de proporção e média com seu desvio padrão com um nível de confiança de 95\%. Para a análise bivariada, utilizou-se 0 teste do qui-quadrado.

0 projeto foi submetido e aprovado pelo Comitê de Ética em Pesquisa da Faculdade de Medicina da Universidade Federal do Ceará.

\section{RESULTAD0S}

Entre 1996 e 2004, no Estado do Ceará, foram registradas 5.105.259 Autorizações de Internação Hospitalar, incluindo 8.399 casos de epilepsia e $425(0,01 \%)$ de cisticercose, sendo 424 de pessoas residentes no Estado do Ceará e 1 no Rio Grande do Norte. 0 ano 2000 foi aquele em que foram

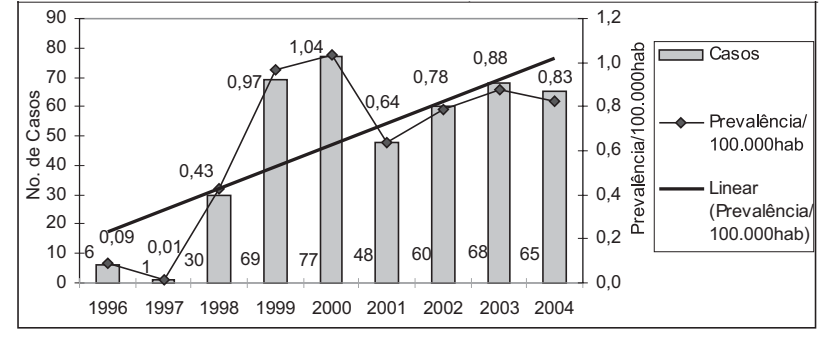

Figura 1 - Número de casos e prevalência de cisticercose, Ceará, 1996 2004.

diagnosticados mais casos (77-18,2\%). Conforme a Figura 1 a tendência é crescente no período $(y=0,986 \mathrm{x}+0,1358)$, com um pico no ano 2000 ( 1,04 caso/100.000hab), voltando a crescer a partir de 2001 (0,64 caso/100.00 hab) com inclinação menor $(\mathrm{y}=0,0665 \mathrm{x}+0,6145)$. Os pacientes foram internados em Fortaleza $(411,96,9 \%)$, Crato (4, 0,9\%), Pedra Branca (3, 0,7\%), Aquiraz, Barro, Cedro, Juazeiro do Norte, Morada Nova, Santa Quitéria ( um caso em cada município, 0,2\%). A Figura 2 mostra que os pacientes com cisticercose eram residentes em 75 (40,8\%) dos 184 municípios cearenses. Entretanto, 273 (69,8\%) residam em

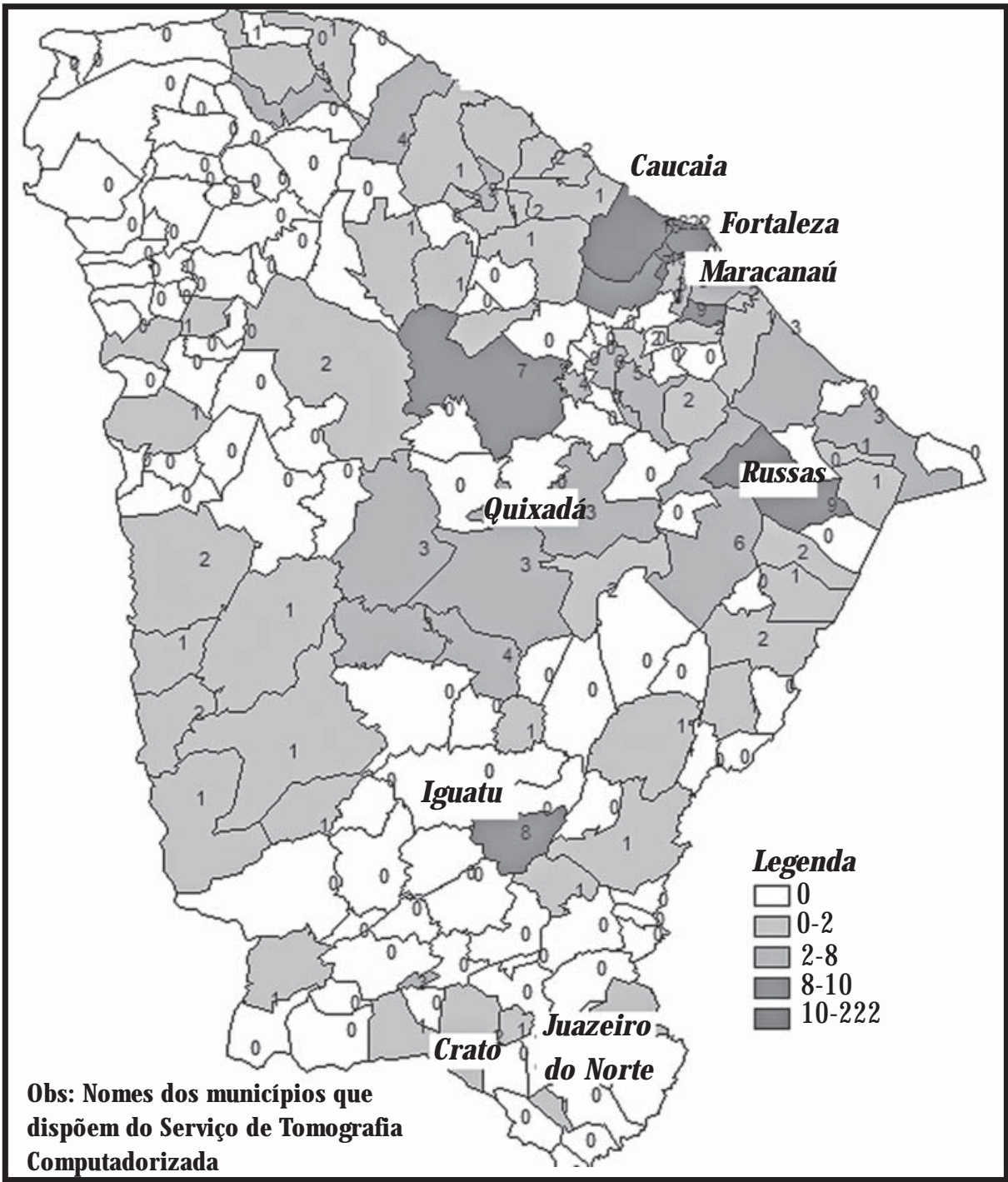

Figura 2 - Distribuição dos casos de cisticercose por município de residência, Ceará, 1996-2004. 
8 municípios: Fortaleza 222 (52,2\%), Maracanaú 16 (3,8\%), Caucaia 15 (3,1\%), Horizonte 9 (2,1\%), Russas 9 (2,1\%), Iguatu $8(1,9 \%)$, Canindé 7 (1,6\%), Maranguape 7 (16\%7). Residiam na região metropolitana de Fortaleza 280 (68,9\%) pacientes. A prevalência variou de 0,05 casos/100.000hab em Juazeiro do Norte a 5,76 em Altaneira, com média de 1,01 casos/100.000hab (DP 0,98). A prevalência anual, considerando todos os municípios do Ceará, variou de 0,01 em 1997 a 1,04 no ano 2000, com média de 0,46 (DP 0,36). Considerando apenas a população dos municípios que registraram casos, a prevalência por 100.000hab variou de 0,02 em 1997 a 1,46 no ano 2000, com média de 0,91 (DP 0,50).

A doença foi diagnosticada em 293 (69,1\%) homens e em 131 (30,9\%) mulheres. Observa-se na Figura 3 que, com exceção dos menores de um ano, houve casos diagnosticados em todas as faixas etárias. A moda foi na faixa de 15 a 19 anos ( 68 casos, prevalência de 0,99 casos/100.000hab) e a mediana na de 30 a 34 anos de idade. A prevalência teve tendência crescente por faixa etária $(y=0,0867 \mathrm{x}+0,0762)$ alcançando 0 máximo de 1,35/100.000hab naqueles de 70 a 79 anos. Os 7 casos de 1996 e 1997 estavam sem classificação da forma clínica, no entanto, foram tratados como neurocisticercose. Dos 417 casos de 1998 a 2004, 410 $(98,3 \%)$ localizavam-se no sistema nervoso central, $2(0,5 \%)$ no olho, $1(0,2 \%)$ em outras regiões e 4 (1\%) não foram especificadas. Dos casos de neurocisticercose, 401 (98,8\%) foram diagnosticadas em Fortaleza, embora apenas $216(52,6 \%)$ residissem nesse município. 0 tratamento foi clínico em 210 (98,7\%) pacientes e cirúrgico em 7 (1,3\%). Evoluíram para o óbito, 4 (1\%) pacientes com neurocisticercose. Nenhum paciente com outras localizações do cisticerco evoluiu para 0 óbito. 0 tempo de permanência foi de menos de 8 dias para $190(45,5 \%)$ pacientes, 7 a 14 dias para 156 $(37,3 \%), 15$ a 21 dias para 50 (12\%), 22 a 28 dias para 14 $(3,3 \%)$ e mais de 29 dias para $8(1,9 \%)$.

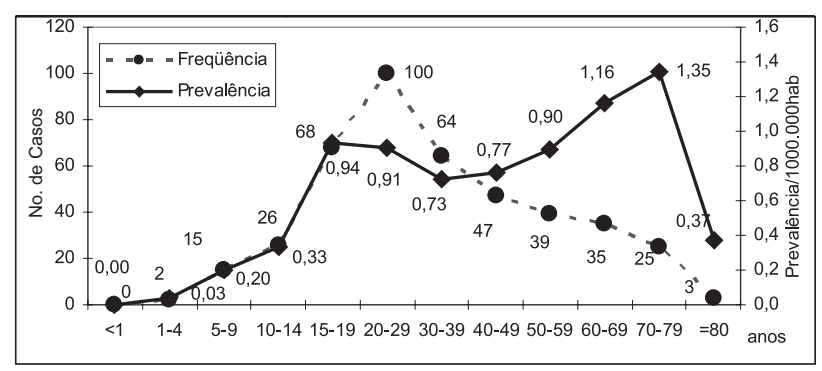

Figura 3 - Número de casos e prevalência de cisticercose por faixa etária, Ceará, 1996-2004.

Nenhum dos municípios com granjas suínas tem prevalência de cisticercose acima da média do estado. Não foi encontrada correlação com 0 número absoluto dos rebanhos suínos registrados pelo $\mathrm{IBGE}^{6} \mathrm{e}$, quando comparada à existência de casos com a razão entre suínos e população do município de residência, verificou-se que os 46 municípios com menor razão suínos/população tinha maior ( $56,5 \%)$ percentual de casos diagnosticados, $(p<0,0061)$ que os 46 municípios com maior (28,3\%) razão suínos/população.

\section{DISCUSSÃ0}

A prevalência de neurocisticercose encontrada neste estudo (1,01 caso/100.000hab) é bem menor que a observada em outros ${ }^{1257910}$. Com exceção de Maracanaú e de Fortaleza, os outros cinco municípios que dispõem de TC não estão entre os que têm mais casos, nem entre os que têm maior prevalência na população. Essa baixa prevalência pode ser 0 resultado da falta de diagnóstico e ter a contribuição da crise da suinocultura no estado. Os pacientes foram internados em apenas nove municípios do estado, em três deles há disponibilidade de tomografia computadorizada (Crato, Fortaleza, Juazeiro do Norte). Essa situação sugere que apenas a existência do tomógrafo no município não foi capaz de estimular o diagnóstico, uma vez que todas as internações de pacientes de Maracanaú, Russas e Quixadá foram feitas em Fortaleza e de Sobral e Barbalha, onde não houve registro de pacientes com cisticercose. 0 SUS é responsável por cerca de $80 \%$ dos atendimentos à população do estado, de modo que o SIH não corresponde à totalidade das internações no estado, o que representa uma limitação, pois reduz o número de casos. Há a possibilidade de erro na codificação da doença, por ocasião da entrada dos dados no $\mathrm{SIH}$, podendo alterar o número de casos no mesmo. Eventualmente, 0 paciente pode ter sido diagnosticado por TCem sua cidade de origem e encaminhado para uma cidade de referência para tratamento. Uma maior cobertura e um melhor registro poderiam tornar esses dados mais representativos do que ocorre na população.

Concluindo, cisticercose é pouco diagnosticada no Ceará, 0 que sugere a necessidade de reforçar sua importância no diagnóstico etiológico de crises convulsivas.

\section{AGRADECIMENTOS}

Fernando Queiroz e Gorete Bezerra da Secretaria Estadual da Saúde do Ceará por complementar as informações sobre o banco de dados SIH-CE.

\section{REFERÊNCIAS BIBLIOGRÁFICAS}

1. Agapejev S. Aspectos clínico-epidemiológicos da neurocisticercose no Brasil. Análise crítica. Arquivos de Neuropsiquiatria 61: 822-828, 2003.

2. Chimelli L, Lovalho AF, Takayanagu OM. Neurocisticercose: contribuição da necrópsia na consolidação da notificação compulsória em Ribeirão Preto-SP. Arquivos de Neuropsiquiatria 56:577-584, 1998.

3. Datasus. Informações de Saúde [on line]. Disponível em http:// www.datasus.gov.br. 2005. Consultado em 27/05/2005.

4. Diário do Nordeste. Campanha quer aumentar 30\% consumo de carne suína no CE. Saúde Animal. Consultado em 26/06/2005. Disponível e m h t t p : / / w w w a grolink. com.br/sa u d a n i mal/ pg_detalhe_noticia.asp?cod=28022, Publicado em 15/06/2005.

5. Gomes I, Veiga M, Correa D. Cysticercosis in epileptic patients of Mulungu do Morro northeastern Brazil. Arquivos de Neuropsiquiatria 58: 621-624, 2002. 
6. Instituto Brasileiro de Geografia e Estatística. Efetivo dos rebanhos. Disponível em: http://www.sidra.ibge.gov.br. Consultado em 31/07/2005.

7. Lino Jr RS, Reis MA, Teixeira VPA. Ocorrência de cisticercose ( Cysticercus cellulosae) encefálica e cardíaca em necropsias. Revista de Saúde Pública 33: 495-498, 1999.

8. Quality Standars Subcommittee of the American Academy of Neurology. Practice parameter: report the utility of neuroimaging in the evaluation of headache in patients with normal neurologic examinations. Neurology, 44:1353-1354, 1994.
9. Rigatti M, Trevisol-Bittencourt PC. Causas de epilepsia tardia em uma clínica de epilepsia do Estado de Santa Catarina. Arquivos de Neuropsiquiatria 57: 787-792, 1999

10. Trevisol-BittencourtPC*, Silva NC, Figueredo R. Neurocisticercose em pacientes internados por epilepsia no Hospital Regional de Chapecó região oeste do Estado de Santa Catarina. Arquivos de Neuropsiquiatria 56: 53-58, 1998.

11. Valença MM, Valença LPAA. Etiologia das crises epilépticas na cidade do Recife, Brasil. Estudo de 249 pacientes. Arquivos Neuropsiquiatria 58: 1064-1072, 2000 